\title{
MINIMUM DISTANCE BASED ROUTING PROTOCOL FOR LIFETIME IMPROVEMENT IN WIRELESS SENSOR NETWORK
}

\author{
Mugdha A. Chaudhari ${ }^{1}$, Sanjay V. Khobragade ${ }^{2}$ \\ ${ }^{I}$ Electronics and Telecommunication Department, Dr. Babasaheb Ambedkar Technological University, Lonere, \\ Raigad, Maharashtra, India \\ ${ }^{2}$ Electronics and Telecommunication Department, Dr. Babasaheb Ambedkar Technological University, Lonere, \\ Raigad, Maharashtra, India
}

\begin{abstract}
Balanced utilization of energy of wireless sensor nodes is a challenge while designing wireless sensor network (WSN). This energy of sensor node is a limited resource and measure for the lifespan of WSN. Communication process consumes most of the energy of sensor node hence; energy of sensor node becomes a major design issue for WSN. Clustering is preferred while designing routing protocols for WSN for its many to one traffic pattern. In our minimum distance based routing protocol for lifetime improvement in WSN (MDBRP) clusters are formed once in a lifetime and their heads are selected rotationally based on minimum communication distance between nodes and their next hop. MDBRP considers minimum energy consumption which aims to increase the overall lifespan of WSN.
\end{abstract}

Keywords - clustering, dynamic clustering, routing protocol, static clustering, wireless sensor network. ****

\section{INTRODUCTION}

Wireless sensor network (WSN) serves in many areas like military surveillance, industrial automation, traffic management, home automation, etc. Various improvements in embedded system technology make it possible to provide low cost, low power, small sized, multifunctional wireless sensor nodes for such broad application areas. Due to the improved features of these wireless sensor nodes over traditional sensors WSN performed very well in various applications mentioned in [1].

Limited battery power of wireless sensor nodes is critical issue while designing and maintaining WSNs. Sensor nodes depletes their energy while establishing routing path for data transfer and mostly in communicating. Communication is most energy consuming task for wireless sensor node [2]. The overall life period of WSN called as lifetime of WSN depends entirely on energy of sensor nodes involved in network. When the energy of sensor nodes goes down to zero or below some threshold value set according to requirement of network then that node is considered as dead. In paper [2] lifetime is defined in two ways such as the time elapsed from the start of network operation to the first death of any node occurred or the time elapsed from the start of network operation to the death of last node in the network. Due to dependence of network's lifetime on energy of sensor nodes it becomes an important design aspect for researchers while designing WSN. Now a day routing protocols are designed so as to balance the energy consumption of sensor nodes to improve lifetime of WSN. MDBRP protocol uses clustering technique for routing characterized by static clusters and dynamic cluster head selection. This protocol insures the minimum communication distance for any node in the network so as to minimize its energy consumption and hence increasing network lifetime.

\section{LITERATURE SURVEY}

A survey about WSN and its applications is done in [3], [4] which elaborates the details of WSN and its parts. Architectural design issues and challenges are explained in [3], [5], [6] which affects the performance of routing protocols. WSN comes under the infrastructureless networks characterized by distributed, low-power and randomly deployed wireless sensor nodes. While developing routing protocol for WSN these aspects have to be considered.

An overview of holes occurring in WSNs is described in [7] stating the causes of creating holes in network. Hole in WSNs is one of the serious design issues. Holes are created when nodes get disabled because of any reason and cause break in the routing path. Hence routing protocol designing considers this as a challenge to overcome through with.

Hierarchical routing protocol based on clustering minimizes the energy consumption of sensor node as in case of LEACH [8]. This protocol operates in terms of round which is considered as the time elapsed for delivery of data from each and every node in the network to the final destination place. Round split into setup phase and steady state phase. During the set up phase cluster heads $(\mathrm{CH})$ are elected among the nodes in network according to threshold set previously. When the heads are elected then remaining nodes decide their respective nearest heads and forms the cluster. After then in steady state phase; actual data transferred to the base station (BS) along with the data fusion at $\mathrm{CH}$.

LEACH-Centralized [9] is derived from LEACH where base station does not allow less energy node to be elected as $\mathrm{CH}$. However in large scale network, nodes far away from BS are unable to send their status to BS. 


\section{NETWORK MODEL}

The network model for this MDBRP protocol assumes all nodes to be homogeneous with respect to their initial energy, sensing capacity and processing ability. All nodes are considered to be stationary, energy constraint and distributed randomly in square field having base station at the very center of field. The BS is assumed stationary with no limited battery power. Every node in the network always has some data to be sent. The network assumes complete data fusion at BS and irrespective of the amount of data received at $\mathrm{CH}$ every node transmits same amount of data. We consider the time elapsed between the start of network to the death of last node in the network as the lifetime of WSN.

\section{MINIMUM DISTANCE BASED ROUTING}

\section{PROTOCOL (MDBRP)}

Proposed MDBRP protocol satisfactorily provides improvement in network lifetime of WSN. Hierarchical clustering is applied in this MDBRP protocol where the clusters remain constant throughout the network operation called as static clustering. The cluster heads are selected rotationally based on minimum communication distance between node and its next hop. Such static clustering assures no coverage holes in network while dynamic $\mathrm{CH}$ selection ensures balanced energy dissipation among the nodes. The data fusion is achieved at the $\mathrm{CH}$ so as to minimize overhead.

\subsection{Formation of Clusters}

The first step of MDBRP protocol is to form clusters by dividing network area after the nodes and BS is deployed randomly. The squared network area is divided into $n$ number of concentric squares. This value of $n$ depends on the distance assumed between two concentric squares $(d)$ which is selected so as to balance the clusters in terms of their area. The value of $n$ can be found from the following equation.

$$
n=\frac{C_{p}(x)}{d}
$$

Where, $\mathrm{C}_{p}(\mathrm{x})$ is $\mathrm{x}$ co-ordinate of center point (BS) of network. Value of $n$ gets increased when network area is expanded. The coordinates of these squares are obtained with the help of distance from the base station to the $n^{\text {th }}$ square is given by following equation

$$
d_{n}=n \times d
$$

Using this distance $d_{n}$ we obtain the coordinates of squares as follows:

$$
\begin{aligned}
& T_{r}\left(S_{n}\right)=\left(C_{p}(x)+d_{n}, C_{p}(y)+d_{n}\right) \\
& T_{l}\left(S_{n}\right)=\left(C_{p}(x)-d_{n}, C_{p}(y)+d_{n}\right) \\
& B_{l}\left(S_{n}\right)=\left(C_{p}(x)-d_{n}, C_{p}(y)-d_{n}\right) \\
& B_{r}\left(S_{n}\right)=\left(C_{p}(x)+d_{n}, C_{p}(y)-d_{n}\right)
\end{aligned}
$$

Where, $\mathrm{S}_{n}$ is $n^{\text {th }}$ square, $\mathrm{T}_{r}$ is top right corner of square, $\mathrm{T}_{l}$ is top left corner of square, $\mathrm{B}_{l}$ is bottom left corner of square, $\mathrm{B}_{r}$ is bottom right corner of square.

In next step the space between two concentric squares is further divided into equal area quadrilaterals as shown in figure 1 denoted as R1 through R9. The inner square is very small in area so kept as a single cluster (R1). Co-ordinates of all regions as shown in figure 1 are obtained using equation below:

$$
\text { Location }=\left(C_{p}(x) \pm d_{x}, C_{p}(y) \pm d_{y}\right)
$$

Where Location is any corner of cluster R1 through R9, $d_{x}$ and $\mathrm{d}_{\mathrm{y}}$ are distances of corner from base station in $\mathrm{X}$ and $\mathrm{Y}$ directions.

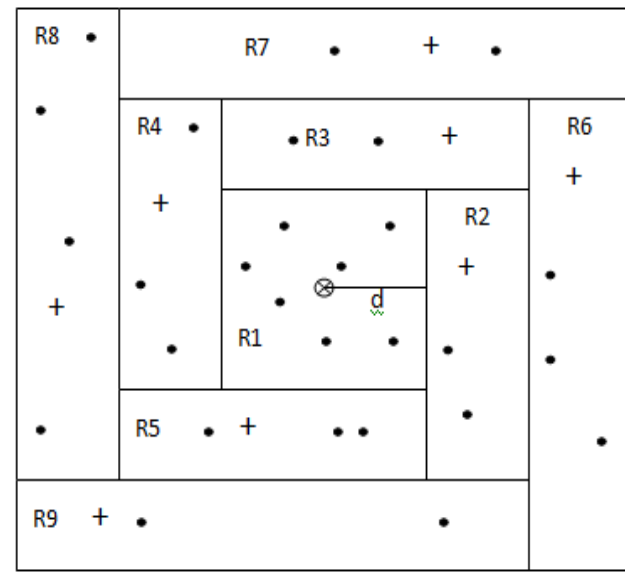

Where,
- Normal Node
+ Cluster Head Node
R1-R9 Clusters
$\otimes$ Base station
$\mathrm{d}$ distance between two squares

Fig. 1 Formation of clusters

\subsection{Cluster Head Selection}

To achieve the equal energy dissipation MDBRP protocol selects $\mathrm{CH}$ at every round. $\mathrm{CH}$ selection is based on minimum communication distance for each node from the reference point of that cluster. The reference point for each cluster is the center of that cluster which is not the same for inner region $\mathrm{R} 1$ as it has minimum area. Nodes in other region (R2 through R9) compare their distance from reference point and the minimum distant node is selected as $\mathrm{CH}$ for the current round. The next minimum distant node is selected as $\mathrm{CH}$ for next round and so on. Nodes in R1 select BS as their next hop.

\subsection{Data Transmission}

In each round of protocol operation first $\mathrm{CHs}$ are selected then data transmission takes place. In this MDBRP protocol communication takes place in two levels of hierarchy. First level communication takes place for regions R1 through R5. The nodes in $\mathrm{R} 1$ send their data directly to base station. $\mathrm{CH}$ of regions R2 through R5 first aggregate their own data along with the data collected from their cluster members and then 
send the fused data to BS. In second level of communication $\mathrm{CHs}$ of region $\mathrm{R} 6$ through $\mathrm{R} 9$ sends their aggregated data to their respective next-hops which are R2 through R5 respectively.

\section{SIMULATION RESULT}

We perform MATLAB simulation for minimum distance based routing protocol and compare our results with that of LEACH protocol. In our network model 120 homogeneous wireless sensor nodes are randomly deployed across square area of $120 \mathrm{~m} \times 120 \mathrm{~m}$ having BS at centre. The radio parameters are taken same as that of LEACH [3], [4]. The packet size is considered of 2000 bits.

We evaluate MDBRP protocol for network lifetime as defined in network model, throughput and average residual energy of network.

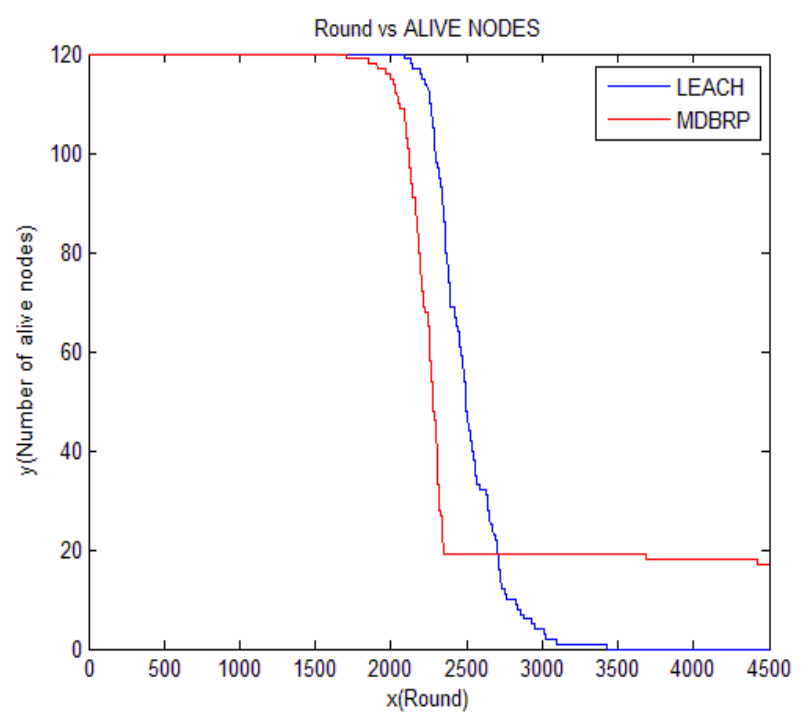

Fig. 2 Network lifetime

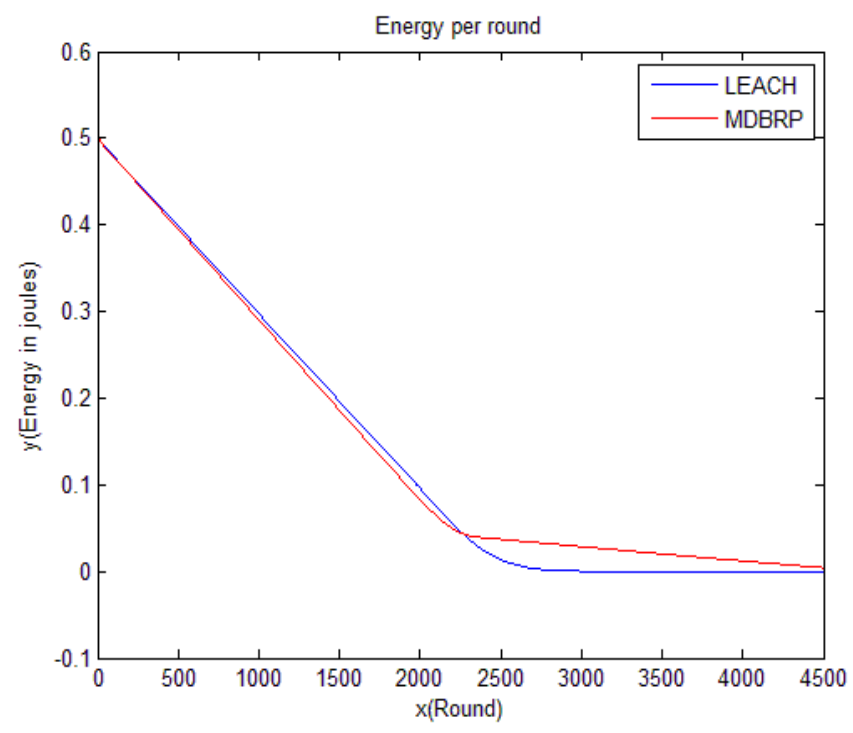

Fig. 3 Residual energy
In figure 2 our protocol has maximum alive nodes as compared to LEACH, which means enhanced lifetime of network also the average residual energy of network can be seen more as compared to LEACH in figure 3. Throughput of the network means number of packets send to base station which are more in case of our MDBRP protocol as in figure 4.

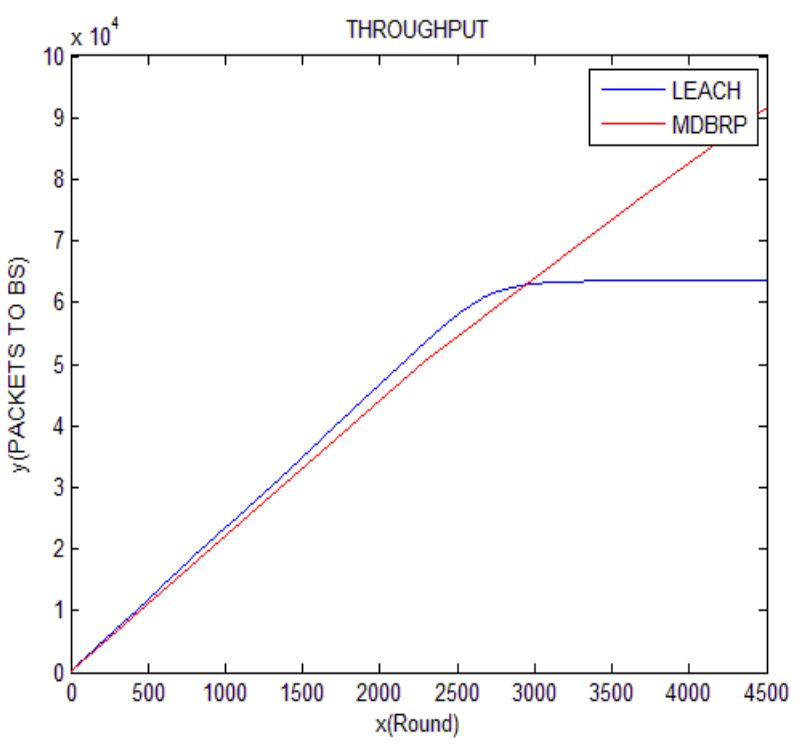

Fig. 4 Throughput

\section{CONCLUSION}

In our work we used hierarchical routing, static clustering and dynamic cluster head selection based on minimum communication distance which will enhance the lifetime of wireless sensor network. Static clustering minimizes the problem of coverage hole by maintaining optimum numbers of clusters. Energy consumption is directly proportional to communication distance hence $\mathrm{CH}$ selection according to minimum communication distance effectively utilizes the limited battery power of sensor nodes. Ultimately this project work will take a step towards satisfying the need of energy efficient architectural routing technique so as to maximize lifetime of wireless sensor network.

\section{REFERENCES}

[1] Mohd Fauzi Othman and Khairunnisa Shazali, "Wireless Sensor Network Applications: A Study in Environment Monitoring System", in International Symposium on Robotics and Intelligent Sensors, by Elsevier, 2012.

[2] Zhao Han, Jie Zhang, Liefeng Liu and Kaiyun Tian, "A General Self-Organized Tree-Based EnergyBalance Routing Protocol for Wireless Sensor Network", IEEE Transactions on Nuclear Science, vol. 61, no. 2, pp. 732-740, April 2014.

[3] Jennifer Yick, Biswanath Mukherjee, Dipak Ghosal, "Wireless sensor network survey", International journal on computer networks by Elsevier, vol. 52, pp. 2292-2330, 2008.

[4] F. Akyildiz, W. Su, Y. Sankarasubramaniam, and E. Cayirci, "Wireless sensor networks: a survey," Computer Networks, vol. 38, no. 4, pp. 393-422, 2002. 
[5] Ajay Jangra and Swati, "Wireless Sensor Network (WSN): Architectural Design issues and Challenges", International Journal on Computer Science and Engineering, vol.02, no.09, pp.3089-3094, 2010.

[6] Sanatan Mohanty, "Energy Efficient Routing Algorithms for Wireless Sensor Networks and Performance Evaluation of Quality of Service for IEEE 802.15.4 Networks", M. Tech. dissertation, National Institute of Technology, Rourkela, India, January, 2010.

[7] Rajat Bhardwaj, Hitesh Sharma, "Holes in wireless sensor networks", International Journal of Computer Science and Informatics (IJCSI), vol-02, Iss-4, pp. 2231-5292, 2012.

[8] Wendi Rabiner Heinzelman, Anantha Chandrakasan, and Hari Balakrishnan, "Energy-Efficient Communication Protocol for Wireless Micro-sensor Networks", in IEEE Proceedings of the 33rd Hawaii International Conference on System Sciences, 2000.

[9] Wendi Rabiner Heinzelman, Anantha Chandrakasan, and Hari Balakrishnan, "An Application-Specific Protocol Architecture for Wireless Micro-sensor Networks", IEEE transactions on wireless communications, vol. 1, no. 4, October 2002. 